\title{
Algoritmos para prevenção e tratamento de lesão por fricção
}

Algorithms for the prevention and treatment of friction injury Algoritmos para prevención y tratamiento de lesión por fricción

\author{
Renan Vinicius Pinheiro ${ }^{1}$ id https://orcid.org/0000-0002-4405-2692 \\ Geraldo Magela Salomé1 io https://orcid.org/0000-0002-7315-4866 \\ Flávio Dutra Miranda' 1 io https://orcid.org/0000-0002-1631-0164 \\ José Ronaldo Alves ${ }^{1}$ io https://orcid.org//0000-0002-4255-8884 \\ Fernanda Aparecida dos Reis ${ }^{1}$ io https://orcid.org//0000-0002-0230-4601 \\ Adriana Rodrigues dos Anjos Mendonça ${ }^{1}$ io https://orcid.org/0000-0003-0526-6636
}

Pinheiro RV Salone $J R$, Reis FA, Mendonça AR. Algoritmos para prevenção e tratamento de lesão por fricção. Acta Paul Enferm. 2021;34:APE03012.

DOI

http://dx.doi.org/10.37689/actaape/2021A003012

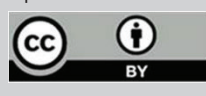

Descritores

Pele; Friç̧ão; Ferimentos e lesões; Avaliação em enfermagem; Algoritmos; Educação em saúde

Keywords

Skin; Friction; Wounds and injuries; Nursing assessment; Algorithms; Health education

Descriptores

Piel; Fricción; Heridas y traumatismos; Evaluación en enfermeira; Algoritmos; Educación en salud

Submetido
4 de Novembro de 2020
Aceito
15 de Junho de 2020

Autor correspondente

Geraldo Magela Salomé

E-mail: salomereiki@univas.edu.br

\section{Resumo}

Objetivo: Elaborar e validar o conteúdo de algoritmos para prevenção e tratamento de lesão por fricção.

Métodos: Para a construção dos algoritmos, foi realizada uma revisão integrativa da literatura após busca nas bases de dados: SciELO, LILACS e MEDLINE. A avaliação dos algoritmos foi realizada por 26 juízes, sendo 10 médicos e 16 enfermeiros, utilizando-se a técnica Delphi. Os resultados foram analisados por meio do Índice de Validade de Conteúdo.

Resultados: No primeiro ciclo de avaliação, os itens dos algoritmos foram considerados pelos juízes de "inadequados" a "totalmente adequados". Após as correções sugeridas pelos juízes, os algoritmos foram reenviados para o segundo ciclo de avaliação, no qual todos os itens foram julgados "adequados" ou "totalmente adequados", resultando em um Índice de Validade do Conteúdo de 1,0.

Conclusão: Os algoritmos desenvolvidos possuem validade de conteúdo e podem auxiliar profissionais de saúde na avaliação, prevenção e tratamento da lesão por fricção.

\section{Abstract}

Objective: Develop and validate the content of algorithms for the prevention and treatment of friction injury.

Methods: For the construction of algorithms, an integrative literature review was conducted after searching SciELO, LILACS and MEDLINE databases. The algorithms were evaluated by 26 raters -10 physicians and 16 nurses - using the Delphi technique. The results were analyzed by the Content Validity Index (CVI).

Results: In the first evaluation cycle, the items of the algorithms were considered "inadequate" to "totally adequate" by the raters. After adjustments suggested by the raters, the algorithms were submitted to a second evaluation cycle, when all items were considered "adequate" or "totally adequate," resulting in a content validity index of 1.0 .

Conclusion: The algorithms have valid content and can help health professionals in the evaluation, prevention and treatment of friction injuries.

\section{Resumen}

Objetivo: Elaborar y validar el contenido de algoritmos para prevención y tratamiento de lesión por fricción.

Métodos: Para la construcción de los algoritmos, se realizó una revisión integradora de la literatura luego de una búsqueda en las bases de datos: SciELO, LILACS y MEDLINE. La evaluación de los algoritmos fue realizada por 26 jueces, de los cuales 10 eran médicos y 16 enfermeros, con la utilización del método Delphi. Los resultados fueron analizados mediante el Índice de Validez de Contenido. 
Resultados: En el primer ciclo de evaluación, los ítems de los algoritmos fueron considerados desde "inadecuados" a "totalmente adecuados" por los jueces. Luego de las correcciones sugeridas por los jueces, se enviaron los algoritmos para el segundo ciclo de evaluación, en el que todos los ítems fueron considerados "adecuados" o "totalmente adecuados", lo que tuvo como resultado un Índice de Validez de Contenido de 1,0.

Conclusión: Los algoritmos elaborados tienen validez de contenido y pueden ayudar a profesionales de la salud en la evaluación, prevención y tratamiento de la lesión por fricción.

\section{Introdução}

A pele é o maior órgão do corpo e está sujeita a agressôes oriundas de fatores patológicos intrínsecos e extrínsecos que podem causar o desenvolvimento de alterações na sua constituição como, por exemplo, lesões por pressão, queimaduras, ulcerações traumáticas, dermatites e lesão por fricção, entre outras, e levar à incapacidade funcional e a mudanças na qualidade de vida dos indivíduos. ${ }^{(1-3)}$ As feridas são eventos comuns no cotidiano dos serviços de atenção à saúde, sendo as mais frequentes a lesão por pressão, úlceras diabéticas, úlceras venosas e lesão por fricção, tornando-se um problema de saúde pública. A presença dessas lesões afeta negativamente a qualidade de vida de indivíduos e de seus familiares, e suas altas taxas de incidência, especialmente na população idosa, provocam impacto econômico na área da saúde. ${ }^{(4,5)}$

O termo em inglês skin tear significa pele (skin) e rasgo ou rompimento (tear). Logo, as lesóes às quais se faz referência neste estudo são abordadas sob a terminologia de lesáo por fricção em virtude da limitação imposta por sua tradução para o português, a qual abarca apenas a fricção em detrimento do cisalhamento (shear) e da contusão (blunt force) como etiologias que também estão relacionadas à ocorrência destas lesóes. ${ }^{(6)}$

A lesão por fricção é resultante de trauma, seja por fricção, contusão ou cisalhamento da pele. A tensão presente na retração, atrito ou choque entre a pele do indivíduo e a superfície do leito ou de objetos ao redor pode provocar feridas de espessura parcial ou de espessura total. A produçáo de exsudato seroso, especialmente nas primeiras 24 horas, torna a lesão por fricção muito úmida. ${ }^{(7,8)}$ Vários estudos relatam que $42 \%$ dessas lesôes se localizam nos cotovelos, $22 \%$ nas pernas e $13 \%$ nas mãos. ${ }^{(9-11)}$

A lesão por fricção ocorre predominantemente entre pessoas idosas ou muito jovens, como neonatos. $\mathrm{O}$ envelhecimento resulta na diminuição das funções da pele e de suas estruturas internas. A pele se torna páli- da, flácida e apresenta menor turgor devido à redução da taxa de renovação celular e da resistência imunológica, entre outros fatores. ${ }^{(12)}$ Estas alterações favorecem a ocorrência de lesão por fricção em idosos.

Os profissionais de saúde que prestam assistência ao idoso desempenham um papel importante no cuidado holístico, o qual considera as necessidades físicas, sociais, emocionais e espirituais do indivíduo, ${ }^{(13)} \mathrm{e}$ fazem um trabalho de extrema relevância na prevenção e no tratamento da lesão por fricção. ${ }^{(14)}$ A equipe de saúde multidisciplinar deve prestar assistência humanizada, sistematizada e personalizada aos idosos com lesão por fricção ou àqueles que apresentam fatores de risco, adotando medidas preventivas ou prescrevendo as coberturas mais adequadas, conforme a categoria da lesão por fricção identificada no exame clínico. ${ }^{(14)}$

O enfermeiro é o profissional que tem maior contato com o paciente, acompanha a evolução da lesão, orienta e executa os cuidados relacionados à prevenção e ao tratamento, e detém o maior domínio das técnicas de cuidado de lesóes em virtude de ter, na sua formação acadêmica, componentes curriculares voltados para esta prática e pelo fato da equipe de enfermagem desenvolvê-las como uma de suas atribuiçôes. ${ }^{(14)}$

A conduta assistencial pode consistir na elaboração de protocolos em forma de algoritmos, os quais devem ser construídos com embasamento científico a partir de revisóes bibliográficas de metanálise de periódicos indexados. Os algoritmos são constituídos por uma sequência finita de instruçóes bem definidas realizadas sistematicamente. São ferramentas primordiais no gerenciamento da qualidade e organização de processos. Em saúde, estes instrumentos são simples, diretos e de fácil acesso; conferem uma visão completa do processo de cuidado, apresentando-se como mapas e servindo de guias para tomadas de decisão. ${ }^{(15-18)}$

Este estudo teve como objetivo elaborar e validar o conteúdo de algoritmos para prevenção e tratamento da lesão por fricção. Esses algoritmos visam fornecer subsídios aos profissionais de saúde que prestam assistência a indivíduos com lesão por fricção ou em 
risco para lesão por fricção para a elaboração e o desenvolvimento de planos de cuidado da lesão com estratégias adequadas de prevenção e tratamento, reunindo uma conduta terapêutica ampla, com variedade de métodos propícios para executá-la.

\section{Métodos}

Estudo aplicado na modalidade de produção de tecnologia, do tipo pesquisa de desenvolvimento metodológico. $\mathrm{O}$ estudo foi aprovado pelo comitê institucional de ética em pesquisa sob o parecer $n^{\circ}$ 1.239.421. o estudo foi realizado no período entre julho de 2016 e agosto de 2017. Todos os profissionais de saúde que concordaram em participar do estudo assinaram o termo de consentimento livre e esclarecido (tcle) ao serem incluídos no painel de juízes. O tcle deixou claro ao participante o teor da pesquisa, garantindo o sigilo das informaçôes pessoais e a livre decisão de retirar, a qualquer momento, o seu consentimento de participação no estudo.

Para a construção dos algoritmos, foi realizada revisão integrativa da literatura junto às bases de dados das ciências da saúde, incluindo o Sistema Online de Busca e Análise de Literatura Médica (MEDLINE), Scientific Eletronic Library Online (SciELO) e Literatura Latino-Americana e do Caribe em Ciências da Saúde (LILACS). Foram utilizados os descritores controlados em ciências da saúde (DeCS): "fricção", "ferimentos e lesões" e "pele", e os termos correspondentes em inglês e espanhol. A estratégia de busca para cada idioma foi determinada pela combinação dos descritores selecionados e o operador booleano " $A N D$ ", conforme os exemplos: (1) fricção $A N D$ ferimentos e lesôes; (2) fricção $A N D$ ferimentos $A N D$ pele.

Os critérios de inclusão para a seleção das publicaçôes foram: apenas estudos primários, publicados nos idiomas português, inglês e espanhol, estar disponível na íntegra e sem delimitação temporal proposta, pois a intenção era compilar todos os estudos que atendessem aos critérios estabelecidos. Foram excluídos: teses, dissertaçôes, monografias, relatórios técnicos, relatos de casos, trabalhos de referência e artigos que, após leitura do resumo, não convergiam com o ob- jeto de estudo proposto, além das publicaçóes que se repetiram nas bases de dados e biblioteca virtual.

A partir deste levantamento, foram elaborados os algoritmos que compreendem uma sequência de procedimentos descrita em quatro etapas: avaliaçáo clínica, classificação da lesão, condutas terapêuticas e medidas preventivas.

A primeira etapa envolve a avaliação clínica. Neste momento, deve ser realizado o exame integral e detalhado das condiçôes da pele do paciente, verificação dos fatores de risco associados que o paciente apresenta para adquirir lesão por fricção, condiçôes da pele adjacente, edema, coloração e aspecto de equimose, presença de retalho de pele, características do retalho (pálido, opaco ou escurecido), presença de sangramento, mensuraçáo, tipo de tecido, presença de exsudato.

A segunda etapa classifica a lesão por fricção. Foi utilizada a versão em português do STAR Skin Tear Classification System. ${ }^{(19)}$ Este instrumento é composto por cinco categorias de lesão por fricção, sendo elas:

- Categoria 1a: lesão por fricção, cujo retalho de pele pode ser realinhado à posição anatômica normal (sem tensão excessiva) e a coloração da pele ou do retalho não se apresenta pálida, opaca ou escurecida.

- Categoria 1b: lesão por fricção cujo retalho de pele pode ser realinhado à posição anatômica normal (sem tensão excessiva) e a coloração da pele ou do retalho apresenta-se pálida, opaca ou escurecida.

- Categoria 2a: lesão por fricção cujo retalho de pele não pode ser realinhado à posição anatômica normal (sem tensão excessiva) e a coloração da pele ou do retalho não se apresenta pálida, opaca ou escurecida.

- Categoria 2b: lesão por fricção cujo retalho de pele não pode ser realinhado à posição anatômica normal (sem tensão excessiva) e a coloração da pele ou do retalho apresenta-se pálida, opaca ou escurecida.

- Categoria 3: lesão por fricção cujo retalho de pele está completamente ausente. ${ }^{(19)}$

A terceira etapa abrange as condutas terapêuticas para tratar a lesão por fricção. Nessa etapa foram apresentados os cuidados locais com a pele e com a lesão, e o curativo ideal que tem como objetivo pro- 
mover a cicatrização da lesão e diminuição da dor. O curativo deve ser facilmente removível e funcionar como uma barreira protetora contra a invasão bacteriana. A escolha da cobertura para a confecção do curativo da lesão por fricção é feita conforme o resultado da avaliação e classificação da lesão.

A quarta etapa apresenta medidas preventivas para lesão por fricção. Após o profissional de saúde realizar a avaliaçáo clínica e a identificação dos fatores de risco intrínsecos e extrínsecos para o desenvolvimento da lesão por fricção, o algoritmo sugere um plano de conduta com medidas preventivas para lesão por fricção.

A validação do conteúdo dos algoritmos foi realizada com médicos e enfermeiros que atuam no tratamento de feridas, enfermeiros pós-graduados em estomaterapia registrados na Associação Brasileira de Estomaterapia, e enfermeiros pós-graduados em dermatologia, registrados na Associação Brasileira de Enfermagem em Dermatologia.

Os critérios de inclusão dos juízes foram: ser graduado em enfermagem ou medicina, com experiência mínima de um ano no tratamento de pacientes com lesóes de pele. Uma carta convite foi enviada para 35 profissionais de saúde. A carta convite incluía a apresentaçáo pessoal inicial e elucidaçóes sobre o tema da pesquisa, o parecer do Comitê Institucional de Ética em Pesquisa, explicaçóes sobre a importância do profissional avaliador para o estudo, um passo-a- passo das etapas para a efetiva participação dos juízes, e a comunicação do prazo de 15 dias para completar o questionário de cada rodada da avaliação e retornar as respostas. Um total de 26 profissionais de saúde aceitaram participar do estudo e retornaram o questionário dentro do prazo solicitado. Os profissionais que aceitaram participar no estudo, mas que não responderam ou retornaram o questionário de avaliação dos algoritmos no prazo de 15 dias foram excluídos.

Para a validação dos algoritmos, foi enviado aos juízes um questionário específico dividido em duas partes: identificaçáo do avaliador, com quatro questôes, e avaliação dos algoritmos, com 15 questóes. Os juízes avaliaram os seguintes temas principais dos algoritmos: conteúdo temático, apresentação gráfica, sequência, clareza e compreensão das informações, definição de lesão por fricção e das categorias, coberturas utilizadas nas diferentes categorias, fatores de risco e condutas preventivas. A escala de Likert foi utilizada nas questóes de avaliação dos algoritmos e tinha como opçóes de respostas: "totalmente adequada", "adequada", "parcialmente adequada", "inadequada” e "não se aplica”. As questôes opcionais de opinião foram mensuradas em escala dicotômica, com respostas "Sim" e "Não", e com instruções para respostas descritivas posteriores.

A técnica Delphi foi usada para a validação dos algoritmos. Esse método tem como característica a obtenção de opinióes de juízes com conhecimento específico em determinada área com o uso de questionários, nos quais os conteúdos dos instrumentos são analisados e julgados pelos juízes em busca de um consenso entre os mesmos. Geralmente são necessários dois ou três ciclos de avaliação, podendo haver mais. ${ }^{(20)}$

O Índice de Validade de Conteúdo foi utilizado para medir o grau de concordância entre os juízes sobre aspectos específicos dos algoritmos desenvolvidos neste estudo, os quais foram examinados por meio do questionário de avaliação. $\mathrm{O}$ valor do Índice de Validade de Conteúdo para a validação de um questionário foi calculado como a soma do número de respostas "adequada" e "totalmente adequada" divido pelo número total de respostas. O valor do Índice de Validade de Conteúdo deve ser maior ou igual a 0,80 (i.e., $80 \%$ de concordância entre os juízes) quando ocorre a participação de seis ou mais juízes na validação do instrumento. ${ }^{(21)}$

\section{Resultados}

Entre os 35 profissionais de saúde convidados para participar do estudo, 26 aceitaram o convite (juízes), sendo 10 médicos e 16 enfermeiros, os quais responderam ao questionário de pesquisa dentro do prazo solicitado de 15 dias.

Os itens dos algoritmos foram avaliados no primeiro ciclo de avaliação como "totalmente adequados" a "inadequados". Embora todos os itens tenham atingido um Î́ndice de Validação de Conteúdo acima do valor mínimo necessário de 0,80 no primeiro ciclo de avaliação, os algoritmos foram revisados com base nas sugestôes apresentadas pelos juízes e 
submetidos a um segundo ciclo de avaliaçáo. Todos os itens dos algoritmos foram avaliados como "totalmente adequados" ou "adequados" no segundo ciclo e, portanto, validados com Índice de Validação de Conteúdo de 1,0, como mostra a tabela 1 .
Após a validação de conteúdo pelos juízes, foram elaboradas as versōes finais dos dois algoritmos. Os esquemas gerais dos algoritmos de prevenção e tratamento de lesão por fricção estáo apresentados nas figuras 1 e 2 , respectivamente.

Tabela 1. Avaliação do conteúdo dos itens dos algoritmos para prevenção e tratamento de lesão por fricção no primeiro e segundo ciclo utilizando-se a técnica Delphi

\begin{tabular}{|c|c|c|c|c|c|c|c|c|c|c|c|}
\hline \multirow{3}{*}{$\#$} & \multirow{3}{*}{ Itens avaliados } & \multicolumn{2}{|c|}{ Inadequada } & \multicolumn{2}{|c|}{$\begin{array}{c}\text { Parcialmente } \\
\text { adequada }\end{array}$} & \multicolumn{2}{|c|}{ Adequada } & \multicolumn{2}{|c|}{ Totalmente adequada } & \multicolumn{2}{|c|}{ IVC } \\
\hline & & $1^{\circ} \mathrm{C}$ & $2^{\circ} \mathrm{C}$ & $1^{\circ} \mathrm{C}$ & $2^{\circ} \mathrm{C}$ & $1^{\circ} \mathrm{C}$ & $2^{\circ} \mathrm{C}$ & $1^{\circ} \mathrm{C}$ & $2^{\circ} \mathrm{C}$ & $1^{\circ} \mathrm{C}$ & $2^{\circ} \mathrm{C}$ \\
\hline & & $\mathrm{N}$ & $\mathrm{N}$ & $\mathbf{N}$ & $\mathrm{N}$ & $\mathrm{N}$ & $\mathrm{N}$ & $\mathrm{N}$ & $\mathrm{N}$ & & \\
\hline 1 & Conteúdo temático & 0 & 0 & 0 & 0 & 10 & 10 & 16 & 16 & 0,932 & 1,00 \\
\hline 2 & Apresentação gráfica & 0 & 0 & 1 & 0 & 6 & 6 & 19 & 20 & 0,962 & 1,00 \\
\hline 3 & Sequência dos algoritmos & 0 & 0 & 3 & 0 & 6 & 6 & 17 & 20 & 0,885 & 1,00 \\
\hline 4 & Clareza e compreensão & 0 & 0 & 3 & 0 & 10 & 11 & 13 & 15 & 0,885 & 1,00 \\
\hline 5 & Definição de lesão por fricção & 0 & 0 & 1 & 0 & 5 & 5 & 20 & 21 & 0,962 & 1,00 \\
\hline 6 & Cuidados antes do curativo & 0 & 0 & 1 & 0 & 5 & 6 & 20 & 20 & 0,962 & 1,00 \\
\hline 7 & Definição das categorias 1a e 1b & 0 & 0 & 1 & 0 & 5 & 6 & 20 & 20 & 0,962 & 1,00 \\
\hline 8 & Tipos de cobertura nas lesões 1a e 1b & 0 & 0 & 1 & 0 & 6 & 6 & 19 & 20 & 0,962 & 1,00 \\
\hline 9 & Definição das categorias 2a e 2b & 0 & 0 & 0 & 0 & 4 & 4 & 22 & 22 & 0,944 & 1,00 \\
\hline 10 & Tipos de cobertura nas lesões 2a e 2b & 0 & 0 & 0 & 0 & 9 & 09 & 17 & 17 & 0,934 & 1,00 \\
\hline 11 & Definição da categoria 3 & 0 & 0 & 0 & 0 & 5 & 4 & 21 & 22 & 0,937 & 1,00 \\
\hline 12 & Tipos de cobertura na categoria 3 & 0 & 0 & 1 & 0 & 9 & 10 & 16 & 16 & 0,962 & 1,00 \\
\hline 13 & Fatores de risco & 0 & 0 & 2 & 0 & 3 & 4 & 21 & 22 & 0,923 & 1,00 \\
\hline 14 & Conduta preventiva com fatores de risco & 0 & 0 & 2 & 0 & 8 & 10 & 16 & 16 & 0,923 & 1,00 \\
\hline 15 & Conduta preventiva sem fatores de risco & 1 & 0 & 3 & 0 & 7 & 8 & 15 & 18 & 0,846 & 1,00 \\
\hline
\end{tabular}

IVC - Índice de Validação de Conteúdo; $1^{\circ} \mathrm{C}$ - primeiro ciclo de avaliação; $2^{\circ} \mathrm{C}$ - segundo ciclo de avaliação; \# - número da questão do questionário de avaliação

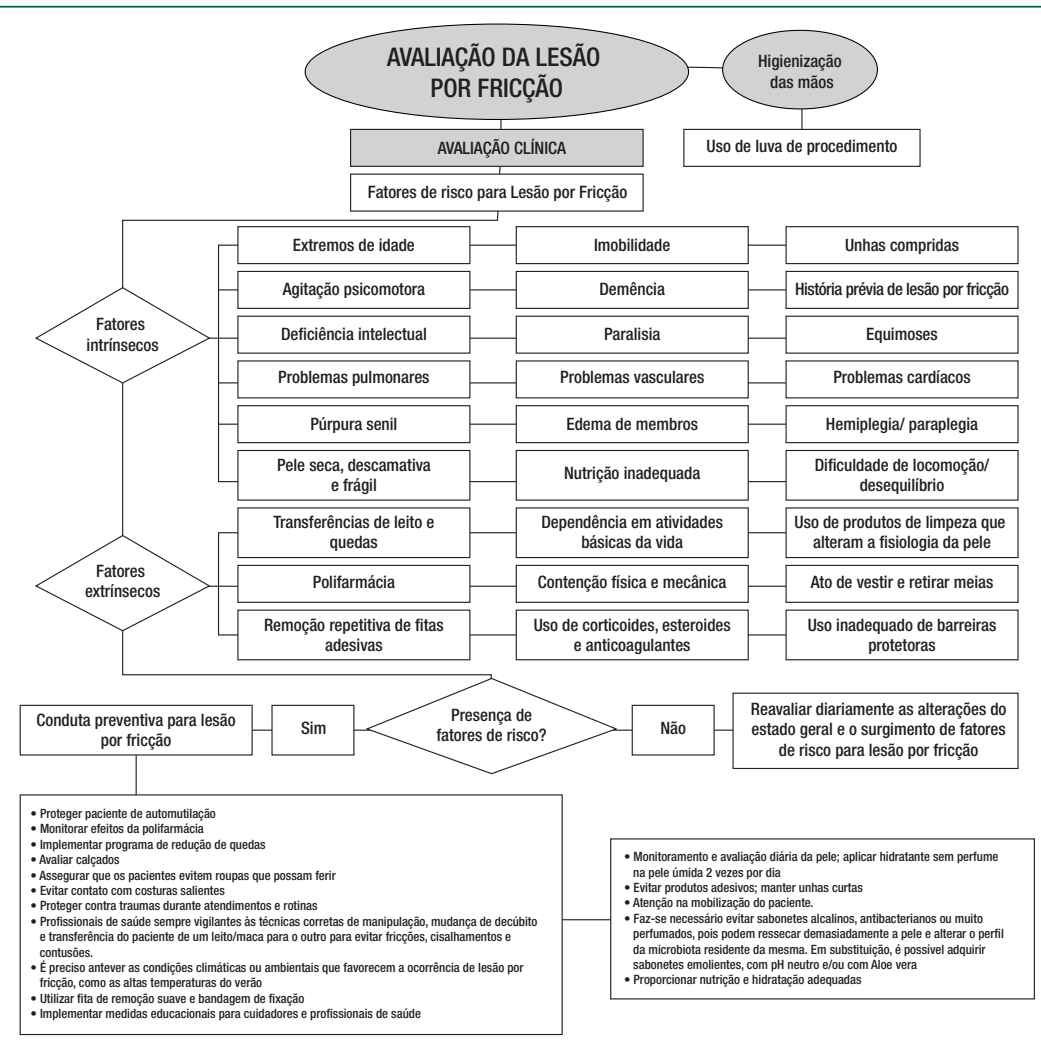

Figura 1. Algoritmo para avaliação de fatores de risco e prevenção de lesão por fricção 


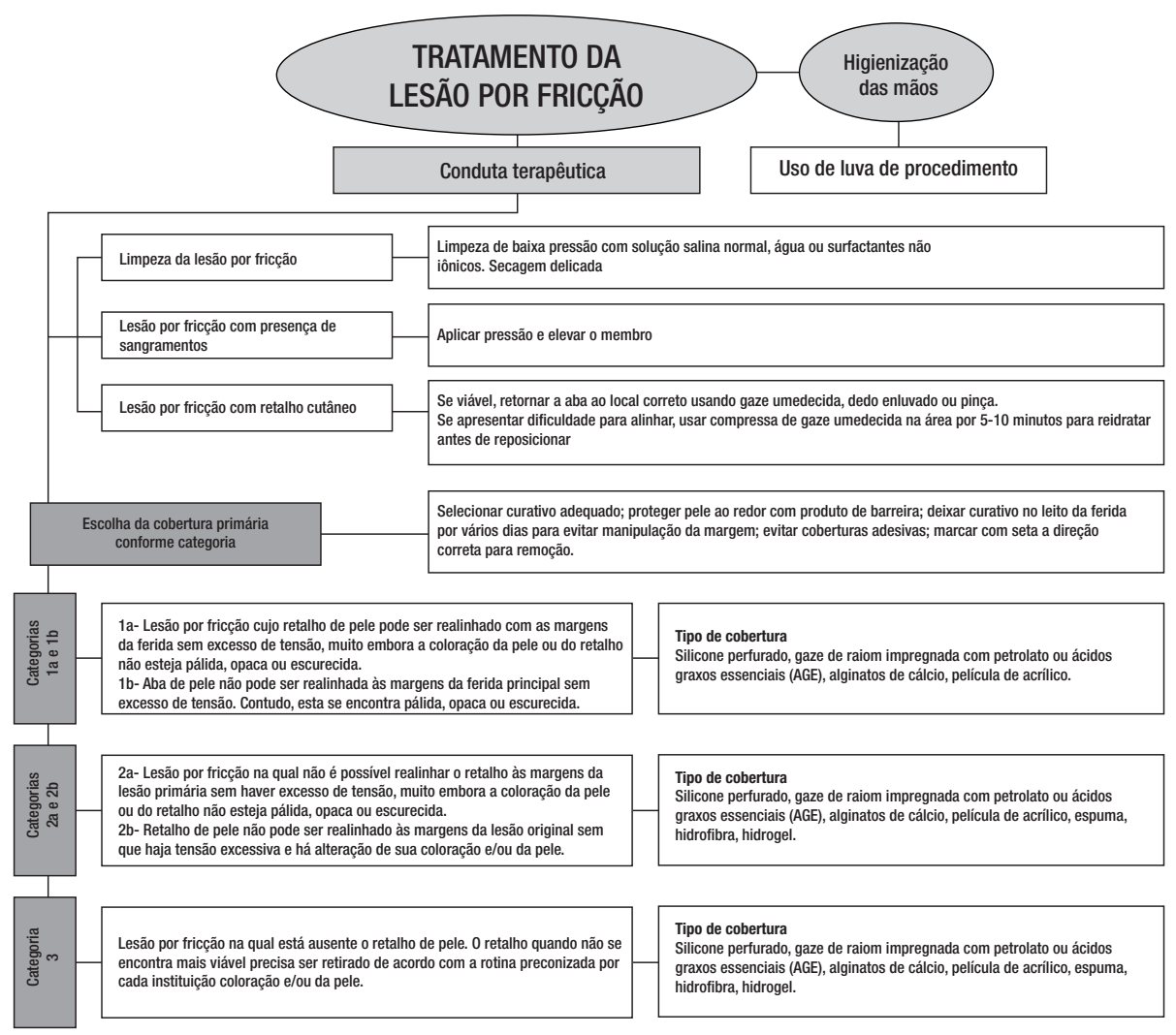

Figura 2. Algoritmo para o tratamento de lesão por fricção

\section{Discussão}

Observa-se um aumento no número de casos de lesão por fricção associado a diversos fatores de risco que estão presentes no cotidiano de pacientes debilitados. Um dos fatores que influenciam o aumento da ocorrência de lesão por fricção é o envelhecimento populacional. Estima-se que cerca de 1,5 milhão de lesôes por fricção ocorram todo ano em idosos institucionalizados. ${ }^{(22-25)} \mathrm{A}$ prevalência da lesão por fricção é três vezes maior que a prevalência de lesão por pressão, com prevalência média de 10,7\%. . $^{(7-23,26)}$

A lesão por fricção impacta diretamente a qualidade de vida devido à possibilidade de ocorrência de infecçôes associadas, as quais aumentam os custos dos cuidados dessa população. $\mathrm{O}$ tratamento adequado da lesão por fricção é fundamental e os materiais selecionados para os curativos devem propiciar uma cicatrização segura, evitando que ocorram outros danos durante o manejo em razão da grande sensibilidade da pele acometida. ${ }^{(25,26)}$

O conhecimento do tema pelos profissionais envolvidos neste tipo de atenção é importante para o sucesso terapêutico. Assim, pensando em facilitar o acesso a essas informaçóes, o presente estudo foi focado no desenvolvimento de algoritmos para prevenção e tratamento de lesão por fricção. Os algoritmos foram construídos após revisão da literatura junto às principais bases de dados de ciências da saúde. A escolha de diferentes coberturas para prevençáo e tratamento de lesóes cutâneas exige dos profissionais de saúde conhecimentos técnicos e científicos relacionados à fisiologia, anatomia, eficácia/efetividade e custo-benefício das coberturas inovadoras existentes no mercado. ${ }^{(3,27)}$

Dois algoritmos foram construídos e validados para conteúdo visando contribuir no gerenciamento e uniformização da assistência de enfermagem ao paciente com lesão por fricção, direcionar a conduta 
e apoiar a decisão do profissional de saúde envolvido na prevenção e tratamento dessa população.

Após o primeiro ciclo de avaliação da versão inicial dos algoritmos desenvolvidos, foram analisadas as respostas e as sugestóes dos juízes referentes a cada questão assinalada como "parcialmente adequada" ou "inadequada”. Essas sugestóes abordavam desde pequenos detalhes, como efetuar a troca de termos para um melhor entendimento do texto, até importantes considerações sobre fatores de risco e mudanças na sequência de apresentaçáo dos itens. Foram acatadas as sugestóes consideradas pertinentes, as quais auxiliaram para que não houvesse respostas negativas no segundo ciclo de avaliação, aumentando a confiabilidade do instrumento final, conforme observado em outros estudos. ${ }^{(7-22,26)}$

Algoritmos construídos para prevenção e tratamento de lesão por fricção devem oferecer ao profissional de saúde a descrição de técnicas, os passos para a execução de determinadas tarefas, informaçóes para o gerenciamento da assistência com qualidade e segurança para o paciente, além de possibilitar ao profissional uma melhor visualização e entendimento do procedimento a ser realizado.

$\mathrm{O}$ índice de validade de conteúdo alcançou um valor próximo a 1,0 em todas as questóes no primeiro ciclo de avaliação e o valor de 1,0 no segundo ciclo, confirmando que todos os aspectos abordados nos algoritmos estão apresentados de forma compreensível e que existiu uma concordância entre os juízes sobre a relevância de todos os itens. Artigos recentes na área de saúde também utilizaram o índice de validade de conteúdo na validação de instrumentos. ${ }^{(27,28,29)} \mathrm{No}$ âmbito da saúde, os algoritmos apresentam uma visão completa do processo de cuidado e apoiam os profissionais de saúde na prestação da assistência com qualidade e em tomadas de decisão, especialmente quando estas são complexas, proporcionando segurança ao paciente. É importante que o instrumento seja validado por profissionais da área de conhecimento do assunto. ${ }^{(15,30)}$

Protocolos, algoritmos, cartilhas, manuais, fluxogramas e diretrizes são considerados ferramentas importantes para o enfrentamento de diversos problemas na assistência e na gestão dos serviços de saúde. Estudos baseados em evidência científica são fundamentados em diretrizes de natureza técnica, organizacional e política, e têm como foco a padro- nização de condutas clínicas, cirúrgicas e preventivas. $^{(31-33)} \mathrm{O}$ desenvolvimento de novas ferramentas requer a incorporação de novas tecnologias que atendam às necessidades de tratamento e das organizaçôes que prestam assistência à saúde. ${ }^{(15,18,31)}$

A cicatrização compreende um complexo processo sistêmico que exige do organismo a ativação, produção e inibição de vários componentes moleculares e celulares. $\mathrm{O}$ algoritmo determina, em sequência ordenada e contínua, o processo de reparação tissular e os fatores de risco para o paciente adquirir uma lesão. Para que haja a otimização da cicatrização da ferida, é imprescindível a manutenção da umidade da lesão. A partir deste preceito, muitas coberturas que favorecem a cicatrização, como as propostas nestes algoritmos, estão disponíveis no mercado para o uso na prevenção e tratamento da lesão por fricção sob a condição de ambiente úmido.

Os algoritmos desenvolvidos têm como propósito nortear os profissionais de saúde a assistir aos pacientes em tempo hábil, de forma efetiva, com qualidade e sem risco e dano ao paciente.

\section{Conclusão}

Nesse estudo foram construídos e validados para conteúdo dois algoritmos que podem auxiliar profissionais de saúde na avaliação, prevenção e tratamento da lesão por fricção.

\section{Colaborações}

Pinheiro RV, Salomé GM, Miranda FD, Alves JR, Reis FA e Mendonça ARA declaram que contribuíram com a concepção do estudo, análise e interpretação dos dados, redação do artigo, revisão crítica relevante do conteúdo intelectual e aprovação da versão final a ser publicada.

\section{Referências}

1. Salomé GM, Ferreira LM. The impact of decongestive physical therapy and elastic bandaging on the control of pain in patients with venous ulcers. Rev Col Bras Cir. 2018 ;45(2):e1385. 
2. Mittag BF, Krause TC, Roehrs H, Meier MJ, Danski MT. Cuidados com lesão de pele: ações da enfermagem. ESTIMA Rev Assoc Bras Estomater. 2017;15(1):19-25.

3. Salomé GM, Ferreira LM. Impact of non-adherent Ibuprofen foam dressing in the lives of patients with venous ulcers. Rev Col Bras Cir. 2017;44(2):116-24.

4. de Almeida SA, Salomé GM, Dutra RA, Ferreira LM. Feelings of powerlessness in individuals with either venous or diabetic foot ulcers. J Tissue Viability. 2014;23(3):109-14.

5. Langemo DK, Williams A, Edwards K. Skin tears: prevention and management. Nursing. 2019;49(4):66-9.

6. Silva CV, Campanili TC, LeBlanc K, Baranoski S, Santos VL. Adaptação cultural e validade de conteúdo do ISTAP Skin Tear Classification para o português no Brasil. ESTIMA. Rev Assoc Bras Estomater. 2018;16:e2618.

7. LeBlanc K, Baranoski S. Skin tears: finally recognized. Adv Skin Wound Care. 2017;30(2):62-3.

8. Skiveren J, Wahlers B, Bermark S. Prevalence of skin tears in the extremities among elderly residents at a nursing home in Denmark. J Wound Care. 2017;26(Suppl 2):S32-6.

9. Rayner R, Carville K, Leslie G, Dhaliwal SS. A risk model for the prediction of skin tears in aged care residents: A prospective cohort study. Int Wound J. 2019;16(1):52-63.

10. LeBlanc K, Baranoski S, Christensen D, Langemo D, Edwards K, Holloway $\mathrm{S}$, et al. The art of dressing selection: A consensus statement on skin tears and best practice. Adv Skin Wound Care. 2016;29(1):32-46.

11. Källman U, Kimberly LB, Bååth C. Swedish translation and validation of the international skin tear advisory panel skin tear classification system. Int Wound J. 2019;16(1):13-8.

12. Tuyama LY, Alves FE, Fragoso MP, Watanabe HA. Feridas crônicas de membros inferiores: proposta de sistematização de assistência de enfermagem a nível ambulatorial. Nursing (São Paulo). 2004;75(7):4650.

13. Gonzaga EL, Navarine TC, Costa MM. Espiritualidade e saúde do idoso: Contribuições para o cuidado holístico de enfermagem. Monteiro SAS, organizador. Políticas de envelhecimento populacional. Ponta Grossa (PR): Atena Editora; 2019.

14. Catunda HL, Bernardo EB, Vasconcellos CT, Moura ER, Pinheiro AK, Aquino PS. Methodological approach in nursing research for constructing and validating protocols. Texto Contexto Enferm. 2017;26(2):e00650016.

15. van Rijswijk L, Beitz JM. Creating a pressure ulcer prevention algorithm: systematic review and face validation. Ostomy Wound Manage. 2013;59(11):28-40.

16. van Rijswijk L, Beitz JM. Pressure ulcer prevention algorithm content validation: a mixed-methods, quantitative study. Ostomy Wound Manage. 2015;61(4):48-57.
17. Stephen-Haynes J. Development of an algorithm as an implementation model for a wound management formulary across a UK health economy. J Wound Care. 2013;22(12):692-8.

18. Metcalf DG, Bowler PG, Hurlow J. A clinical algorithm for wound biofilm identification. J Wound Care. 2014;23(3):137-8.

19. Strazzieri-Pulido KC, Santos VL, Carville K. Cultural adaptation, content validity and inter-rater reliability of the "STAR Skin Tear Classification System”. Rev Lat Am Enfermagem. 2015;23(1):155-61.

20. Cassiani SH, Rodrigues LP. A técnica de Delphi e a técnica de grupo nominal como estratégias de coleta de dados das pesquisas em enfermagem. Acta Paul Enferm. 1996;9(3):76-83.

21. Wynd CA, Schmidt B, Schaefer MA. Two quantitative approaches for estimating content validity. West J Nurs Res. 2003;25(5):508-18.

22. Serra R, lelapi N, Barbetta A, de Franciscis S. Skin tears and risk factors assessment: a systematic review on evidence-based medicine. Int Wound J. 2018;15(1):38-42.

23. Mclnulty L. Prevention and management of skin tears in older people. Emerg Nurse. 2017;25(3):32-9.

24. Benbow M. Assessment, prevention and management of skin tears. Nurs Older People. 2017;29(4):31-9.

25. Salcido R. Falls or skin tears: which came first? Adv Skin Wound Care. 2016;30(1):6.

26. Idensohn P, Beeckman D, Campbell KE, Gloeckner M, LeBlanc K, Langemo D, et al. Skin tears: a case-based and practical overview of prevention, assessment and management. J Community Nurs. 2019;33(2):32-41.

27. Carvalho MR, Salomé GM, Ferreira LM. Construction and validation of algorithm for treatment of pressure injury. J Nurs UFPE online. 2017;11(10):4171-83.

28. Salomé GM, Bueno JC, Ferreira LM. Multimedia application in a mobile platform for wound treatment using herbal and medicinal plants. J Nurs UFPE online. 2017;11(Supl 11):4579-88.

29. Cunha DR, Salomé GM, Massahud MR Junior, Mendes B, Ferreira LM. Development and validation of an algorithm for laser application in wound treatment. Rev Lat Am Enfermagem. 2017;25(0):e2955.

30. da Silva Torres F, Blanes L, Freire Galvão T, Masako Ferreira L. Development of a manual for the prevention and treatment of skin tears. Wounds. 2019;31(1):26-32.

31. Candeloro G. Skin tears. A quantitative study on the phenomenon and proposal for a prevention and management procedure. Ital J Wound Care. 2017;1(2):61-6.

32. Santos AC, Dutra RA, Salomé GM, Ferreira LM. Construction and internal reliability of an algorithm for choice cleaning and topical therapy on wounds. J Nurs UFPE online. 2018;12(5):1250-62.

33. Salomé GM, Ferreira LM. Developing a mobile app for prevention and treatment of pressure injuries. Adv Skin Wound Care. 2018;31(2):1-6. 